\title{
HOMOGENEOUS DIELECTRIC HEATING IN LARGE MICROWAVE OVENS BY EXCITATION OF MULITIPLE EIGENMODES AT THEIR RESONANCE FREQUENCIES
}

\author{
Dominik Neumaier $^{1}$, Sabrina Sanseverino ${ }^{1}$, Guido Link ${ }^{1}$ and John Jelonnek ${ }^{1,2}$ \\ $I H M^{1}, I H E^{2}$, Karlsruhe Institute of Technology (KIT), 76131 Karlsruhe, Germany \\ dominik.neumaier@kit.edu
}

Keywords: homogeneous microwave heating, mode analysis, industrial microwave heating

\begin{abstract}
Microwave heating faces growing interest in many industries because of its large potential in increasing productivity and energy efficiency. Nevertheless, the main challenge of this heating method is to provide sufficient uniformity of the temperature distribution, in particular in industrial scale production. Several temperature maxima and minima typically occur in a sample because of the standing waves inside a cavity. An optimization-based approach is presented to increase the temperature homogeneity by exiting selected eigenmodes of the cavity. Results of a related experiment show the performance of the developed algorithm.
\end{abstract}

\section{Introduction}

Dielectric heating by microwaves offers several advantages if comparing to conventional heating. One major advantage is the volumetric heating effect. It is mostly independent from the heat conduction of the work piece. In addition, selective microwave heating is possible. It just depends on the selected loss factors of the processed materials and results in temperature profiles beneficial for specific applications. Hence, the microwave heating can significantly improve the cycle time in production and the energy efficiency in the industrial heating process. The main challenge is to achieve a sufficient temperature uniformity in the product exposed to the electromagnetic field profile in the microwave oven.

Several research activities concerning this topic were already reported. In [1] and [2] microwave applicators with magnetrons as RF (radio frequency) sources are presented. By using magnetrons, it is easily possible to change the amplitude and the pulse duration of the RF power during the heating process. On the other hand, a frequency or phase tuning of magnetrons is only limited possible if not at all impossible practically. In this frame in [1] and [2], an intelligent control algorithm is used to control the amplitudes of the different magnetrons to increase the temperature uniformity of the work piece. In contrast to the magnetron, power modules that are using solid-state amplifiers (SSA) would offer the possibility to vary accurately the amplitude, frequency and phase of the RF output power. In [3], using a simulative approach, it is shown that an intelligent change in frequency will improve the homogeneity of the temperature distribution inside a work piece. In addition, a phase shift between multiple microwave sources used can further improve the temperature homogeneity [4]. In contrast to both mentioned publications, an optimization algorithm is proposed which aims for the homogenous heating by changing both, the frequency and the amplitude of the SSA. To show this, an industrial scale cavity with a well-designed antenna is used which fulfills the following requirements: 
1. measurement the ratio of the reflected power to the transmitted power of the antenna during the process to detect the resonance frequencies,

2. amplitude (power) and frequency tuneability of the SSA,

3. direct comparison of experimental results with simulations.

\section{Experimental Setup}

Fig. 1 shows the multimode microwave oven used in the experiment. It has an industrial scale of $535 \mathrm{~mm} \times 510 \mathrm{~mm} \times 395 \mathrm{~mm}$. The corners are rounded and some deformations of the cavity exist to mount the sample holder. By using the analytical model from [5] and by assuming that the microwave oven is a perfect rectangular cavity exactly 32 eigenmodes exist in the $2.45 \mathrm{GHz}$ ISM band. For the measurement of the temperature distribution a sheet of paper $(510 \mathrm{~mm} \times 380 \mathrm{~mm})$ was used as the dielectric load. It was placed on a very low loss PTFE plate as a sample holder. The advantage of the used load is that the heating patterns can be illustrated very well, especially because the paper has a very low thermal conductivity.

In addition, for the purposed microwave cavity, an antenna system was developed based on numerical simulations with CST Microwave Studio. A loop antenna was designed (Fig. 1 (right)) and the positions of four loop antennas were optimized to excite at least $90 \%$ of those 32 eigenmodes of the unloaded cavity. In the following measurement system only the antenna, which excites most eigenmodes, was installed.

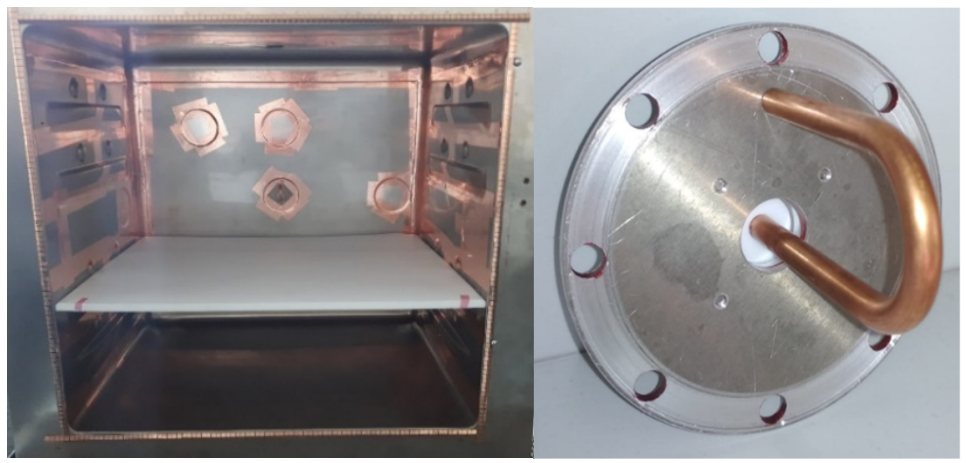

Fig. 1 Cavity geometry with a PTFE plate as a sample holder (left). Designed loop antenna (right).

Fig. 2 shows the schematic measurement setup. Firstly, the setup is capable to identify the 32 resonance frequencies of the eigenmodes that exist in the ISM Band from $2.4 \mathrm{GHz}$ to $2.5 \mathrm{GHz}$. Secondly, it allows the measurement of the power ratio of the reflected to transmitted power of the antenna while using the newly developed $300 \mathrm{~W}$ tunable RF power modules from $\mathrm{HBH}$ microwave GmbH (Germany). The RF power modules operate in the ISM frequency range from $2.4 \mathrm{GHz}$ to $2.5 \mathrm{GHz}$. The RF power modules can be used as amplifiers that are driven by a low-power signal or as free-running RF generators at a specific RF output power. It is possible to do very fast and precise frequency steps $(10 \mathrm{kHz}$ step size, $1 \mathrm{~ms}$ switching time). The RF output power is adjustable in $10 \mathrm{~W}$ steps (also PWM is supported which provide 1000 steps and 10 ns pulses). Between the antenna and the SSA a directional coupler and a circulator are located. The circulator is integrated in the main housing of the RF power module. It protects the amplifier from power reflected at the entrance of the cavity that may affect or in worst case destroy the SSA. Even more, by using the directional coupler, type Microlab CK-19N it is possible to record the reflected power. The $S_{21}$ measurement of the vector network analyzer (VNA) R\&S ZVL enables the 
measurement of the resonance frequencies of the cavity by analyzing the ratio between the reflected and transmitted power. A calibration of the directional coupler and RF power module is not necessary because only the resonance frequency is important and not the magnitude of it. At the top side of the cavity, an IR camera is installed. It allows to observe the temperature distribution of the load during the heating process. A computer is used for a fully automated system control and data recording.

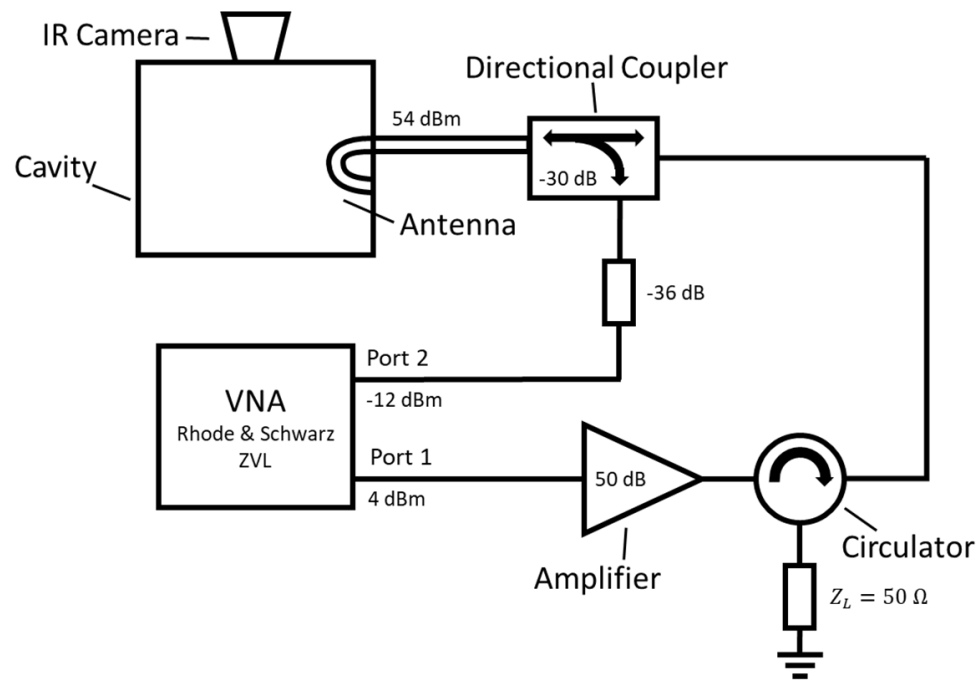

Fig. 2 Measurement setup.

The design of the loop antenna and the positioning in the cavity was done with the help of CST Microwave Studio. In this paper, only one antenna is used. The purposed system enables a later operation with up to four antennas in parallel. Fig. 3 exemplary illustrates the comparison of the simulated power distribution with the measured temperature distribution for two representative eigenmodes. It is possible to identify different high order modes of the simulation in a real measurement. The frequency shift between the measured and simulated resonance frequencies of less than $1 \%$, which appears, may be explained with geometrical inaccuracies in the simulation model. 


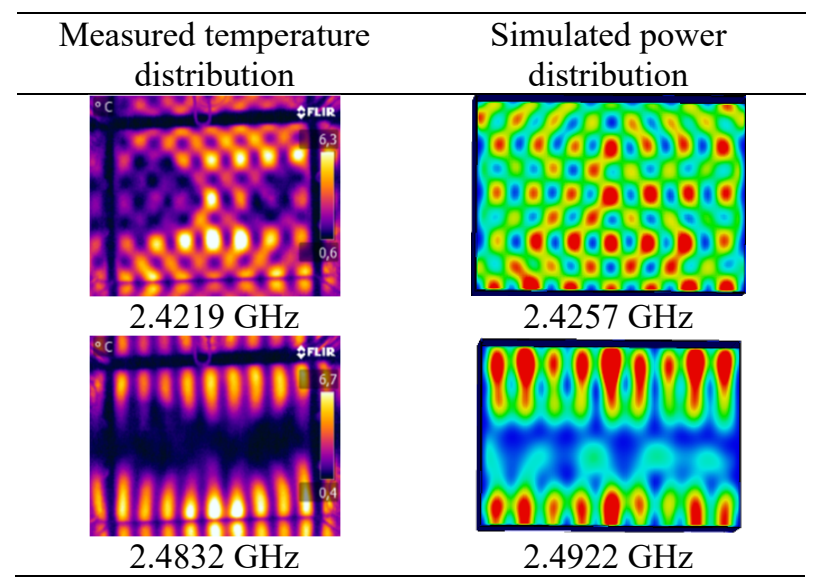

Fig. 3 Comparison of simulated with measured modes.

\section{Methods}

In following a method is described to increase the temperature homogeneity by superimposing different eigenmodes, each with its individual heating pattern. The idea is to switch very fast between different modes. Instead of changing the amplitude only the time, in which each mode is active, changes. The advantages of this is that nonlinearities of the $\mathrm{RF}$ power module do not need to be considered and it can be operated with maximum efficiency.

Fig. 4 presents the approach of the used algorithm for superimposing the eigenmodes. At first a measurement of $S_{21}$ is done in the frequency range of $2.4 \mathrm{GHz}$ to $2.5 \mathrm{GHz}$. By searching the minima of the $S_{21}$ spectrum, all resonance frequencies can be detected. The easiest way to detect how much energy the load absorbs at those eigenmodes is the measurement of the temperature increase of the load by exciting each mode the same time duration. Every resonance frequency is excited for $20 \mathrm{~s}$ and after that, the sample cooled down. The temperature difference between the start (sample is cold) and end (after $20 \mathrm{~s}$ ) temperature distribution is recorded. Every resonance frequency couples a different power in the load and therefore there is a different temperature change. To increase comparability of every eigenmode the recorded thermal images $\Delta T(x, y)$ were normalized as follows:

$$
\Delta \mathrm{T}_{\text {norm }}(\mathrm{x}, \mathrm{y})=\Delta T(x, y) / \max _{x, y}[\Delta T(x, y)],
$$

where $x$ and $y$ describe the coordinates of the picture in pixel. 


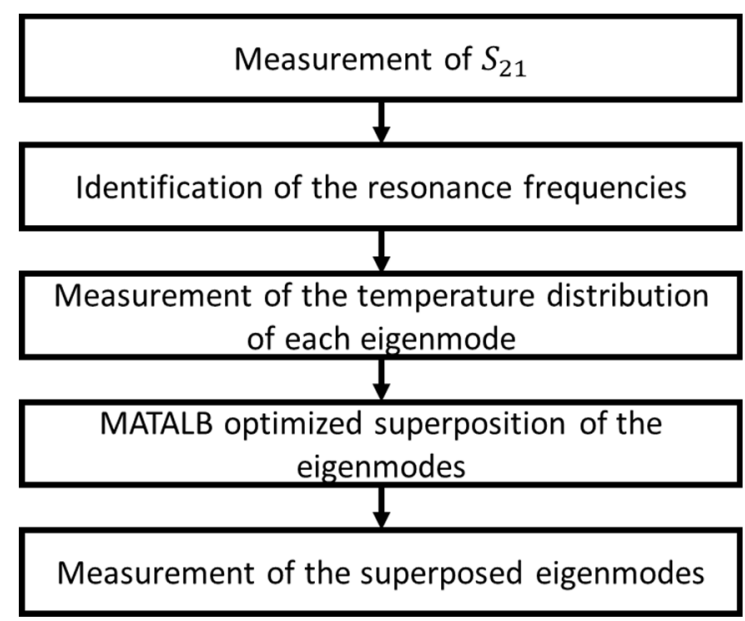

Fig. 4 Approach for superimposing the eigenmodes.

By using an optimizer, the optimal superposition of the different modes is calculated. The aim is to calculate a homogeneous superposition of the eigenmodes. Therefore the objective function

$$
J(\boldsymbol{k})=\max _{x, y}\left[\sum_{i=1}^{N}\left(k_{i} \cdot \Delta \mathrm{T}_{\mathrm{i}}(x, y)\right)\right]-\min _{x, y}\left[\sum_{i=1}^{N}\left(k_{i} \cdot \Delta \mathrm{T}_{\mathrm{i}}(x, y)\right)\right]
$$

is chosen. It describes the difference between the maximum of $\Delta T$ and the minimum of $\Delta T$ of the superimposed image. The value

$$
\boldsymbol{k}=\left[k_{1}, k_{2}, \ldots, k_{N}\right], k_{i} \in \mathbb{R}
$$

is a parameter which specifies the time duration every mode $i$ is exited. $N$ represents the number of considered eigenmodes. The optimization task

with the constraints

$$
\min _{\boldsymbol{k}}(J(\boldsymbol{k}))
$$

$$
\begin{gathered}
k_{i} \geq 0, \\
\sum_{i=1}^{N} k_{i}=1
\end{gathered}
$$

is solved in MATLAB. Only with the constraints it is guaranteed that the optimizer will not find a negative $k_{i}$, which is comparable with cooling the sample. In addition, the sum over all $k_{i}$ must be something else than zero otherwise the optimum would be zero. By choosing this value to 1 , the percentage of each used eigenmode can directly calculated with $k_{i}$.

\section{Results}

The optimization method is tested with the described measurement setup. About 30 different resonance frequencies were excited, including that modes which does not couple significant RF power into the cavity. Therefore, only eigenmodes are used for optimization, which effect at least a $3{ }^{\circ} \mathrm{C}$ temperature change after $20 \mathrm{~s}$ heating.

In Fig. 5 the normalized heating patterns of all eigenmodes are presented which are used by the optimizer and have a $k_{i} \neq 0$.

The optimizer chooses eigenmodes so that hotspots are at maximum different positions. 


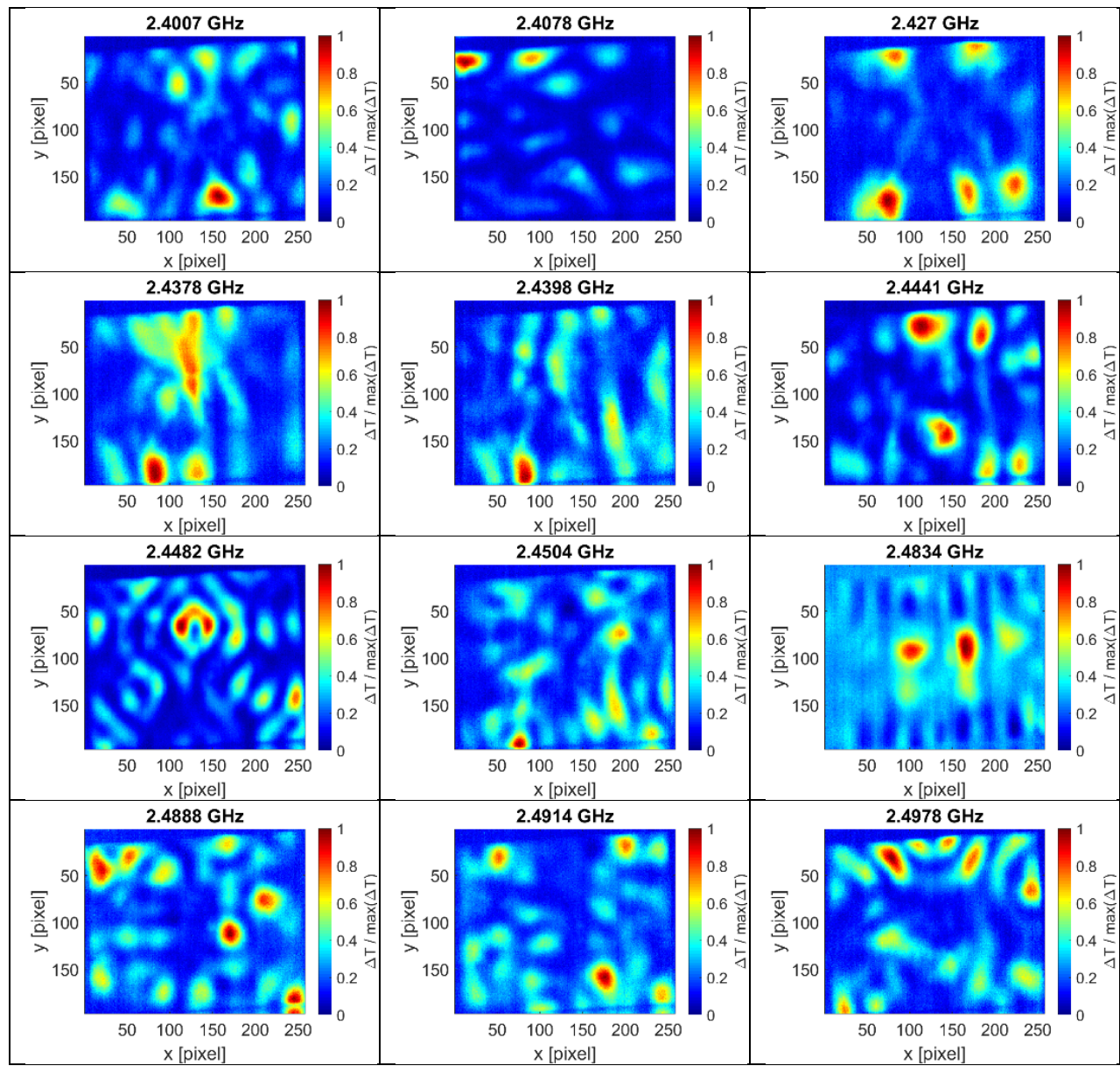

Fig. 5 Eigenmodes used by the optimizer.

The MATLAB optimized solution is presented in Fig 6 (left). The temperature uniformity with the optimized superposition is much higher than for each individual eigenmode (see Fig. 5). Thus, the optimized solution by MATLAB as described before was verified with the given setup. The period for sweeping through all modes is $0.5 \mathrm{~s}$. Every resonance frequency is excited with a time duration corresponding to its $k_{i}$. The measured results are demonstrated in Fig 6 (right). The MATLAB optimized image and the measured image are very similar to each other.
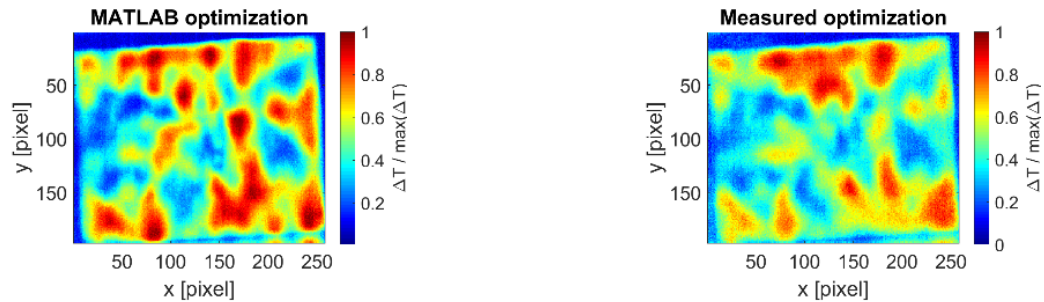

Fig 6. In MATLAB optimized superposition (left). Measured optimization (right). 
For a better comparison a criteria for homogenous heating is defined. At first, all images are normalized like in Fig. 5 and Fig 6. Then the standard deviation

$$
s=\sqrt{\frac{1}{N_{x} \cdot N_{y}-1} \sum_{x=1}^{N_{x}} \sum_{y=1}^{N_{y}}\left[\Delta \mathrm{T}_{\text {norm }}(\mathrm{x}, \mathrm{y})-1\right]^{2}}
$$

is generated for each point in the picture. $N_{x}$ and $N_{y}$ represent the number of pixels in $x$ - and $y$-direction. Instead of using the mean value as a reference, the value 1 is used. Otherwise, also a picture, which has nearly everywhere cold spots, would also create good results.

In Table 1 the results of the defined criteria are listed. All eigenmodes at their resonance frequency have a much higher standard deviation in comparison with the optimized solution. Furthermore, the results of the MATLAB optimization and in the real measurement are nearly the same.

Table 1. Criteria for homogenous heating for each eigenmode and the optimized solution.

\begin{tabular}{|c|c|c|}
\hline Frequency [GHz] & Standard deviation $\boldsymbol{s}$ & ${\text { Variance } \boldsymbol{s}^{\mathbf{2}}}^{\mathbf{G}}$ \\
\hline 2.4007 & 0.8194 & 0.6715 \\
\hline 2.4078 & 0.8716 & 0.7597 \\
\hline 2.4270 & 0.7892 & 0.6229 \\
\hline 2.4378 & 0.7520 & 0.5654 \\
\hline 2.4398 & 0.7758 & 0.6018 \\
\hline 2.4441 & 0.8081 & 0.6530 \\
\hline 2.4482 & 0.8234 & 0.6780 \\
\hline 2.4504 & 0.7682 & 0.5901 \\
\hline 2.4834 & 0.7061 & 0.4986 \\
\hline 2.4888 & 0.7541 & 0.5687 \\
\hline 2.4914 & 0.7627 & 0.5817 \\
\hline 2.4978 & 0.7647 & 0.5848 \\
\hline MATLAB optimization & 0.5352 & 0.2865 \\
\hline Measured optimization & 0.5522 & 0.3049 \\
\hline
\end{tabular}

In addition, a synthetic leather is used instead of the sheet of paper as a sample. On top of the leather, there is some glue, which has higher dielectric losses (Fig. 7 (left)). In this case, also the MATLAB optimization and the measured optimization deliver nearly the same results (Fig. 7).
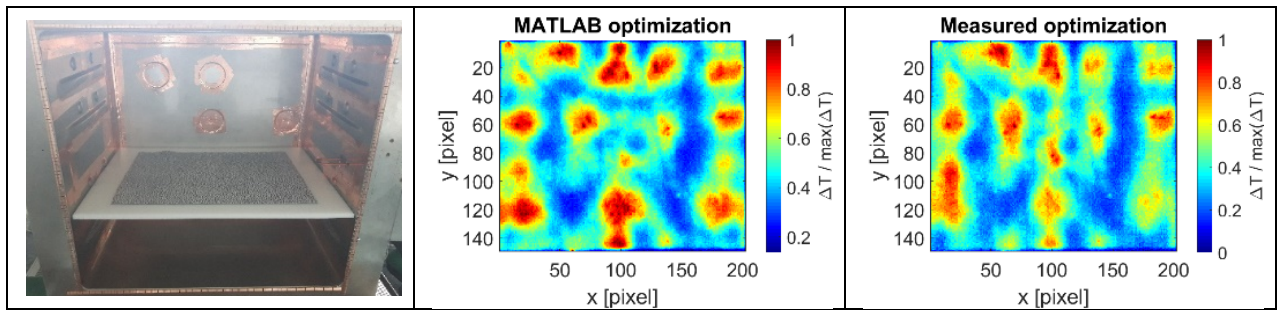

Fig. 7 Measurement of a synthetic leather with some glue on top (left). MATLAB optimization (middle). Measured optimization (right). 


\section{Conclusion and Outlook}

In this paper, an approach is demonstrated which allows to increase the homogeneity of the temperature distribution of a flat sample, that is heated by microwaves from a well controlled RF power module. At first, a setup has been designed that allows detecting and precisely characterizing available eigenmodes in the microwave applicator. Furthermore, a CST model was developed that describes the real experiment. In a first approach by use of a single antenna only, the uniformity of heating has increased. To make use of the full potential of this technique in a next step multiple antennas will be used to get access to more eigenmodes and therefore to increase the degrees of freedom. In addition, a parallel activation of different antennas is possible. The final objective is the implementation of this method into a temperature control algorithm.

\section{Acknowledgment}

The authors acknowledge the financial support by the Federal Ministry for Economic Affairs and Energy of Germany in the project ZF4204602PR6.

\section{References}

[1] Sun, Y., Adaptive and Intelligent Temperature Control of Microwave Heating Systems with Multiple Sources, PhD thesis, KIT Scientific Publishing , 2016. doi:10.5445/KSP/1000051503.

[2] Zhou, J., Li, Y., Li, N., Liu, S., Cheng, L., Sui, S., and Gao, J, A multi-pattern compensation method to ensure even temperature in composite materials during microwave curing process, Composites Part A: Applied Science and Manufacturing, vol. 107, pp. 10-20, 2018. doi: 10.1016/j.compositesa.2017.12.017

[3] Yakovlev V. V, Frequency control over the heating patterns in a solid-state dualsource microwave oven, in 2015 IEEE MTT-S International Microwave Symposium, pp. 1-4, 2015. doi: 10.1109/mwsym.2015.7166948

[4] Gambato, F, Moro, F. and Guarnieri, M., Improving microwave cooking performance by source phase shifting, in IMPI's 48th Annual Microwave Power Symposium (IMPI 48), vol. 48. Mechanicsville, VA: International Microwave Power Institute, 2014, pp. 26-29.

[5] Pozar, D. M., Microwave Engineering, 4th ed. Hoboken: Wiley, 2012 